\title{
Non-linear, continuous-wave EPR spectroscopy and spin-lattice relaxation: spin-label EPR methods for structure and dynamics $\uparrow$
}

\author{
Derek Marsh, ${ }^{*, a}$ Vsevolod A. Livshits ${ }^{b}$ and Tibor Páli ${ }^{c}$ \\ ${ }^{a}$ Max-Planck-Institut für biophysikalische Chemie, Abt. Spektroskopie, Am Fassberg 11, \\ D-37077 Göttingen, Germany \\ ${ }^{b}$ Institute of Chemical Physics, Russian Academy of Sciences, 117977, Moscow, \\ Russian Federation \\ ${ }^{c}$ Institute of Biophysics, Biological Research Center, H-6701 Széged, Hungary
}

\begin{abstract}
The sensitivity of continuous-wave, non-linear EPR signals to spin-lattice $\left(T_{1}\right)$ relaxation has been investigated. The aim was to identify those spectral displays that are most appropriate to obtain structural and dynamic information from spin-label EPR experiments that involve detection of $T_{1}$-relaxation enhancements. This has been achieved by solving the Bloch equations for the various harmonics of the absorption and dispersion components of the spin magnetisation. Of interest are the magnetisation components that are out-of-phase with respect to the static magnetic field modulation, under conditions of partial saturation of the microwave absorption. It is found that both the first- and second-harmonic out-of-phase absorption EPR signals are particularly sensitive to $T_{1}$-relaxation. The first-harmonic absorption quadrature-phase signal is favoured for determining $T_{1}$-relaxation enhancements because of its superior intensity and relative insensitivity to transverse $\left(T_{2}\right)$ relaxation. The second-harmonic absorption quadrature-phase EPR signal has lower relative intensity and is more sensitive to $T_{2}$-relaxation, but has a unique sensitivity to rotational diffusion that is exploited in saturation transfer EPR spectroscopy. The non-linear dispersion signals are less appropriate because of their principal sensitivity to $T_{2}$-relaxation and because they saturate less readily. These novel non-linear EPR spectroscopies can be contrasted with the conventional progressive saturation behaviour of the in-phase absorption signals that are determined by the $T_{1} T_{2}$ relaxation time product.
\end{abstract}

\section{Introduction}

Spin-lattice $\left(T_{1}\right)$ relaxation enhancement of stable aminoxyl free radicals used as spin labels has proved to be a sensitive means for studying slow exchange processes (both physical/ chemical exchange and Heisenberg spin exchange) and also weak dipolar and spin exchange interactions with paramagnetic ions (see e.g. refs. 1,2). Such spin-label EPR experiments are capable of yielding both structural and dynamic information about biomembranes and other biological systems (e.g. ref. 3), particularly when combined with the molecular genetic technique of site-directed spin-labelling on selective cysteine-mutant proteins. ${ }^{4}$ Continuous-wave saturation EPR experiments with magnetic field modulation have proved useful for measurements on biological systems because of their relative simplicity, accessibility and high signal sensitivity. In addition to conventional progressive saturation studies, continuous-wave EPR spectroscopy that involves detection of the non-linear response in phase quadrature with the magnetic field modulation offers an alternative and potentially more sensitive approach. It has been demonstrated that the non-linear second-harmonic, absorption saturation-transfer EPR spectroscopy, which is normally used for studying slow rotational diffusion, ${ }^{5}$ may be used successfully also for spin-label applications involving spin-lattice relaxation enhancement (e.g. refs. 6, 7).

In the present paper, the sensitivity of the various non-linear EPR displays to spin-lattice relaxation is investigated from solution of the Bloch equations for the spin magnetisation by including the effects of the static field modulation, in addition

$\uparrow$ Presented at the 30th International Meeting of the Electron Spin Resonance Group of the RSC, University of Lancaster, 6-10th April 1997.

† Formerly known as nitroxide. to those of the microwave magnetic field. ${ }^{8}$ The various harmonics of the absorption and dispersion signals that are out-ofphase with respect to the field modulation are considered, under conditions of partial saturation. The aim is to identify those non-linear EPR displays that are most sensitive to $T_{1^{-}}$ relaxation, as opposed to rotational diffusion, and also that are least affected by other processes, in particular transverse $\left(T_{2}\right)$ relaxation. It is predicted that the dispersion signals are less appropriate for such studies than are the harmonics of the out-of-phase absorption signals. In particular, attention is drawn to the first-harmonic, absorption, quadrature-phase EPR signal. This non-linear display is unsuitable for the more usual saturation transfer EPR studies of slow rotational diffusion, but is found to be appropriate for studies of $T_{1}$-relaxation enhancement. Special advantages of the first-harmonic nonlinear spectra are the relatively high signal intensity, the predicted insensitivity to $T_{2}$-relaxation, and the ability to tune the range of $T_{1}$-relaxation rates to which the spectral intensities are sensitive by varying the modulation frequency, whilst still maintaining adequate signal strengths.

\section{Theoretical background}

It is necessary to determine the non-linear EPR signals that are obtained in phase quadrature to the static magnetic field modulation, $H_{\mathrm{m}} \cos \omega_{\mathrm{m}} t$, under conditions of partial saturation of the microwave power absorption. This is achieved by solution of the standard Bloch equations for the spin magnetisation, $\boldsymbol{M}$, taking explicit account of the microwave and modulation fields, $H_{1}$ and $H_{\mathrm{m}}$, in addition to the longitudinal $\left(T_{1}\right)$ and transverse $\left(T_{2}\right)$ relaxation processes.

The Fourier harmonics, $n$, of the spin magnetisation with respect to the field modulation frequency, $\omega_{\mathrm{m}}$, are given by eqn. (1), ${ }^{9}$ where $\boldsymbol{M}=M_{0}(u, v, w)$ is the spin magnetisation 


$$
\boldsymbol{M}=\sum_{n=-\infty}^{\infty} \boldsymbol{M}_{n} \mathrm{e}^{-\mathrm{i} n \omega_{\mathrm{m}} t}
$$

expressed in a coordinate frame rotating at the Larmor frequency about the static field direction, which is specified by the $z$-axis. Solution of the Bloch equations for the spin magnetisation in the rotating frame is obtained by expanding the Fourier amplitudes, $\boldsymbol{M}_{n}$, in a power series of the field modulation amplitude, $k$ eqn. (2), ${ }^{8}$ where $k=H_{\mathrm{m}} / 2 \Delta H$ is the

$$
M_{n}=\sum_{v=0}^{\infty} M_{n, v} k^{v}
$$

modulation amplitude, $H_{\mathrm{m}}$, normalised with respect to the linewidth, $\Delta H$. The expansion coefficients $\boldsymbol{M}_{n, v}=\left(u_{n, v}, v_{n, v}, w_{n, v}\right)$ are non-zero only for $v \geqslant n$. To lowest order in the field modulation amplitude, $k$, the harmonics of the absorption and dispersion EPR signals that are in phase quadrature with respect to the field modulation are given, respectively, by eqns. (3) and (4),

$$
\begin{aligned}
& v_{n}{ }^{\prime}=k^{n} \operatorname{Im}\left(v_{n, n}\right) \\
& u_{n}{ }^{\prime}=k^{n} \operatorname{Im}\left(u_{n, n}\right)
\end{aligned}
$$

where the components of $\boldsymbol{M}_{n, v}$ are obtained by substituting eqns. (1) and (2) in the Bloch equations that contain specifically the microwave and modulation fields, $H_{1}$ and $H_{\mathrm{m}}$, respectively. ${ }^{7}$ The harmonics of the conventional EPR spectrum that are inphase with the field modulation are given by corresponding expressions involving the real part of $\boldsymbol{M}_{n, v}$. The spectral lineshapes and integrated intensities are obtained from the explicit dependence of the transverse components of $\boldsymbol{M}_{n, v}$ on the static magnetic strength, $H_{0}$.

\section{Results and discussion}

\section{Model calculations: sensitivity to $T_{1}$ and $T_{2}$}

Analytical expressions are obtained, to the lowest order in modulation amplitude, for the spectral amplitudes of the various harmonics of the out-of-phase absorption and dispersion signals $\left(v_{n}{ }^{\prime}\right.$ and $u_{n}{ }^{\prime}$, respectively) under partial microwave saturation (see refs. 7, 8). In each case, terms involving the saturation parameter, $\gamma_{\mathrm{e}}^{2} H_{1}^{2} T_{1} T_{2}$ where $\gamma_{\mathrm{e}}$ is the electron gyromagnetic ratio are retained, but the reasonable approximations that $T_{2} / T_{1} \ll 1$ and $\omega_{\mathrm{m}}{ }^{2} T_{2}^{2} \ll 1$ are made. The zeroth harmonic solutions, $v_{0,0}$ and $u_{0,0}$ are identical to those for the conventional 'slow passage' Bloch equations. They are independent of the field modulation amplitude and have no out-of-phase components.

The amplitudes of the first-harmonic, out-of-phase absorption and dispersion signals are given respectively by eqns. (5) and (6), where the amplitude of the dispersion signal $u_{1}{ }^{\prime}(0)$ is

$$
\begin{gathered}
v_{1}{ }^{\prime}(\Delta H)=-\frac{1}{2} \gamma_{\mathrm{e}} H_{\mathrm{m}} \frac{\gamma_{\mathrm{e}} H_{1} \omega_{\mathrm{m}} T_{2}^{3}}{1+\frac{1}{2} \gamma_{\mathrm{e}}{ }^{2} H_{1}{ }^{2} T_{1} T_{2}} \times \\
\frac{\gamma_{\mathrm{e}}{ }^{2} H_{1}^{2} T_{1}^{2} /\left(1+\omega_{\mathrm{m}}{ }^{2} T_{1}^{2}\right)-1}{\left[2+\gamma_{\mathrm{e}}{ }^{2} H_{1}{ }^{2} T_{1} T_{2} /\left(1+\omega_{\mathrm{m}}{ }^{2} T_{1}^{2}\right)\right]^{2}} \\
u_{1}{ }^{\prime}(0)=\frac{1}{2} \gamma_{\mathrm{e}} H_{\mathrm{m}} \frac{\gamma_{\mathrm{e}} H_{1} \omega_{\mathrm{m}} T_{2}^{3}}{1+\gamma_{\mathrm{e}}{ }^{2} H_{1}^{2} T_{1} T_{2}}
\end{gathered}
$$

measured at the centre of the line, and that of the absorption signal $v_{1}{ }^{\prime}(\Delta H)$ is measured at a distance equal to the intrinsic (zeroth harmonic) linewidth, $\Delta H=1 / \gamma_{\mathrm{e}} T_{2}$, from the centre. (The amplitude at the centre of the line is zero, in the latter case.) From the structure of eqns. (5) and (6), it is clear that the out-of-phase absorption signal is far more sensitive to $T_{1}$ relaxation than is the dispersion signal. Apart from the saturation factor in the denominator of eqn. (6), the maximum amplitude of the dispersion signal is dependent only on $T_{2}$ relaxation.
The amplitude of the second-harmonic, out-of-phase absorption is given by eqn. (7). (This spectral display corres-

$$
\begin{aligned}
& v_{2}{ }^{\prime}(0)=-\frac{1}{4} \gamma_{\mathrm{e}}{ }^{2} H_{\mathrm{m}}{ }^{2} \frac{\gamma_{\mathrm{e}} H_{1} \omega_{\mathrm{m}} T_{2}{ }^{4}}{1+\gamma_{\mathrm{e}}{ }^{2} H_{1}{ }^{2} T_{1} T_{2}} \times \\
& {\left[\frac{\gamma_{\mathrm{e}}^{2} H_{1}^{2} T_{1}\left(2 T_{1}+5 T_{2}\right)}{1+2 \gamma_{\mathrm{e}}^{2} H_{1}^{2} T_{1} T_{2}+4 \omega_{\mathrm{m}}^{2} T_{1}^{2}}-3\right]}
\end{aligned}
$$

ponds to the standard second-harmonic saturation transfer spectroscopy.) Again, as for the first-harmonic, the secondharmonic out-of-phase absorption signal possesses an additional sensitivity to $T_{1}$, beyond that expressed simply by the saturation factor. The first and second-harmonic non-linear absorption EPR spectra therefore possess a superior sensitivity to $T_{1}$-relaxation processes than do conventional progressive saturation experiments performed on the in-phase spectra. The $T_{1}$-sensitivity of the latter is determined solely by the saturation factor (see e.g. ref. 10). For the second-harmonic absorption out-of-phase spectra, the enhanced sensitivity to $T_{1}$-relaxation enhancement has previously been demonstrated experimentally. 6,11

For experimental analysis, the non-linear out-of-phase signals are conveniently normalized to the conventional firstharmonic in-phase spectral intensity, $v_{1}(\Delta H)$. This gives an internal standard to correct for differences in number of spins, spectrometer gain and detection sensitivity, etc. (see e.g. ref. 12). The corresponding normalized intensities for the first- and second-harmonic out-of-phase absorption signals therefore take the forms given by eqns. (8) and (9), where it is assumed for

$$
V_{1}^{\prime}(\Delta H) \sim \omega_{\mathrm{m}} T_{2} \frac{\gamma_{\mathrm{e}}^{2} H_{1}^{2} T_{1}^{2} /\left(1+\omega_{\mathrm{m}}{ }^{2} T_{1}^{2}\right)-1}{\left[2+\gamma_{\mathrm{e}}^{2} H_{1}^{2} T_{1} T_{2} /\left(1+\omega_{\mathrm{m}}^{2} T_{1}^{2}\right)\right]^{2}}
$$

$V_{2}^{\prime}(0) \sim \frac{1}{2} \gamma_{\mathrm{e}} H_{\mathrm{m}} \omega_{\mathrm{m}} T_{2}^{2}\left[\frac{\gamma_{\mathrm{e}}^{2} H_{1}^{2} T_{1}\left(2 T_{1}+5 T_{2}\right)}{1+2 \gamma_{\mathrm{e}}^{2} H_{1}^{2} T_{1} T_{2}+4 \omega_{\mathrm{m}}{ }^{2} T_{1}^{2}}-3\right]$

simplicity that the conventional in-phase spectral intensity of the first-harmonic varies as $v_{1} \sim\left(\frac{1}{2} \gamma_{\mathrm{e}}^{2} H_{\mathrm{m}} H_{1} T_{2}^{2}\right) /\left(1+\gamma_{\mathrm{e}} H_{1}^{2} T_{1} T_{2}\right)$. The latter condition is deduced from the real part of the expansion coefficients of the spin magnetisation obtained in the solution of the Bloch equations. As a first approximation, solely for the purposes of the normalisation, the weaker dependence of the in-phase signal on modulation frequency is ignored.

Expressions (8) and (9) may be used to explore the sensitivity of the first- and second-harmonic non-linear spectra to the $T_{1}$ and $T_{2}$-relaxation times. The predicted dependences of the normalised spectral intensity on spin-lattice relaxation for various values of $T_{2}$ are given in Figs. 1 and 2 for the first-harmonic and second-harmonic signals, respectively. These model calculations indicate that both the first-harmonic and the secondharmonic out-of-phase spectral amplitudes are sensitive to the spin-lattice relaxation time over a wide range of $T_{1}$-values. This range includes values that are of relevance in spin-label EPR experiments. $^{3,5,7}$ Over an appreciable region the normalised amplitude depends approximately linearly on the $T_{1}$-relaxation time, as found experimentally for the second-harmonic out-ofphase signal in response to spin-label relaxation enhancements by paramagnetic ions or spin-spin interactions. ${ }^{7,13,14}$

In order to obtain sensitivity of the first-harmonic quadrature signal to a wide range in $T_{1}$, it is necessary to use a somewhat lower modulation frequency, $\omega_{\mathrm{m}}$, than for the secondharmonic quadrature signal. However, because of the intrinsically higher intensity of the first-harmonic signal, intensities comparable to those of the second-harmonic signal are obtained, in spite of the lower modulation frequency (compare Figs. 1 and 2). At higher modulation frequencies, the firstharmonic quadrature signal is sensitive to shorter values of $T_{1}$, i.e. to faster spin-lattice relaxation rates. Comparison of Figs. 1 


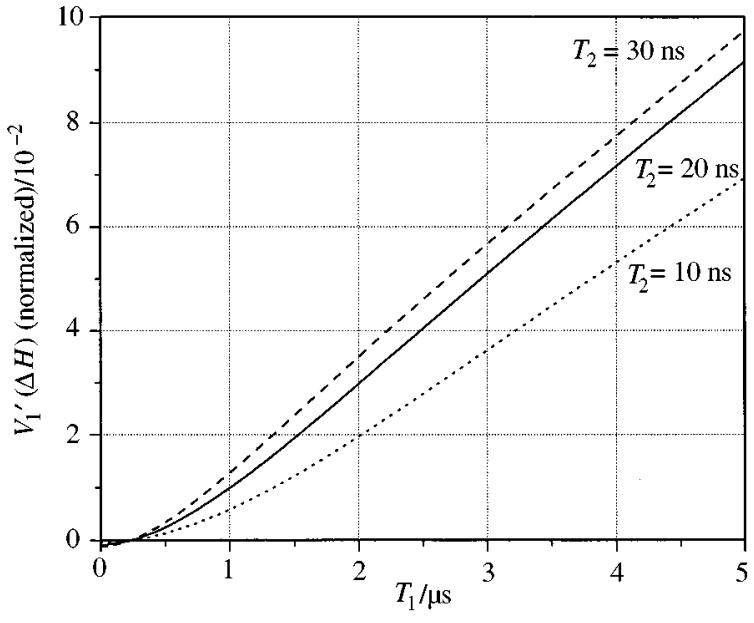

Fig. 1 Dependence of the normalized amplitude of the first-harmonic absorption out-of-phase EPR signal, $\quad V_{1}{ }^{\prime}(\Delta H)=v_{1}{ }^{\prime}(\Delta H) \times$ $\left[\left(1+\frac{1}{2} \gamma_{\mathrm{e}}{ }^{2} H_{1}{ }^{2} T_{1} T_{2}\right) /\left(\frac{1}{2} \gamma_{\mathrm{e}}{ }^{2} H_{\mathrm{m}} H_{1} T_{2}{ }^{2}\right)\right]$, on $T_{1}$-relaxation time for various values of $T_{2}$. The normalized amplitude is calculated from eqn. (8) with $\omega_{\mathrm{m}} / 2 \pi=2.5 \times 10^{4} \mathrm{~Hz}, H_{1}=0.25 \mathrm{G}$ and the values of $T_{2}$ indicated on the figure.

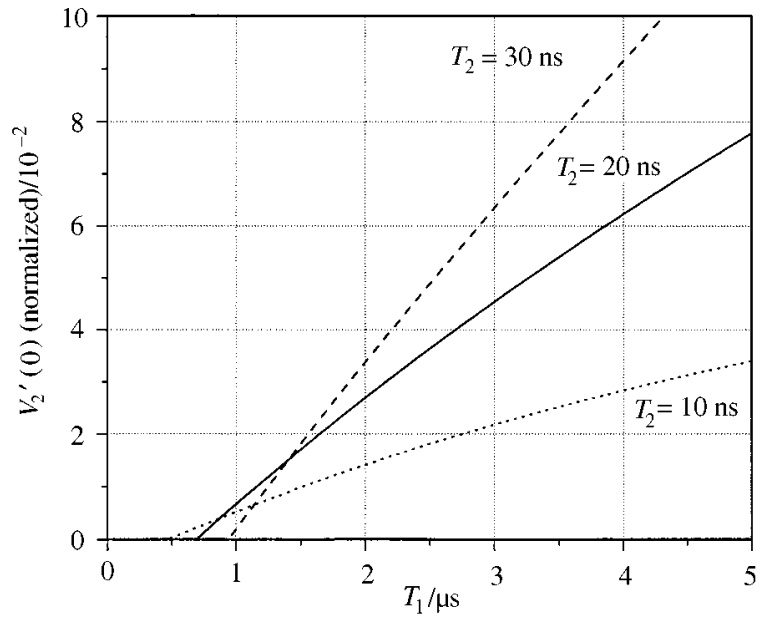

Fig. 2 Dependence of the normalized amplitude of the secondharmonic absorption out-of-phase EPR signal $V_{2}^{\prime}(0)=v_{2}{ }^{\prime}(0)[(1+$ $\left.\left.\gamma_{\mathrm{e}}^{2} H_{1}^{2} T_{1} T_{2}\right) /\left(\frac{1}{2} \gamma_{\mathrm{e}}^{2} H_{\mathrm{m}} H_{1} T_{2}^{2}\right)\right]$, on the $T_{1}$-relaxation time for various values of $T_{2}$. The normalized amplitude is calculated from eqn. (9) with $\omega_{\mathrm{m}} / 2 \pi=5 \times 10^{4} \mathrm{~Hz}, H_{1}=0.25 \mathrm{G}, H_{\mathrm{m}}=5 \mathrm{G}$ and the values of $T_{2}$ indicated on the figure.

and 2 indicates that the first-harmonic quadrature signal is far less sensitive to the transverse relaxation time, $T_{2}$, than is the second-harmonic quadrature signal. This is of advantage for estimating absolute values of $T_{1}$, and favours use of the firstharmonic quadrature absorption signal for $T_{1}$-sensitive experiments. However, for relative $T_{1}$-relaxation enhancements, in the absence of appreciable changes in $T_{2}$, the second-harmonic quadrature absorption signal may equally be used. Currently, most non-linear experiments have been performed with the second-harmonic display (for a review, see ref. 3). In contrast, the second-harmonic display is far more sensitive to anisotropy in the relaxation rates, and hence to rotational diffusion, as is exploited in the standard saturation transfer EPR experiment. ${ }^{5}$ The lineshape of the first-harmonic quadrature absorption signal, however, is relatively insensitive to rotational diffusion and therefore is unsuitable for the conventional application of saturation transfer EPR in studying slow rotational diffusion.

\section{Applications and simulations}

In practice, the non-linear saturation transfer EPR spectra are normally analysed in terms of the integrated spectral intensities. ${ }^{7,12}$ This method of analysis circumvents potential problems arising from inhomogeneous broadening, ${ }^{10}$ and in the case

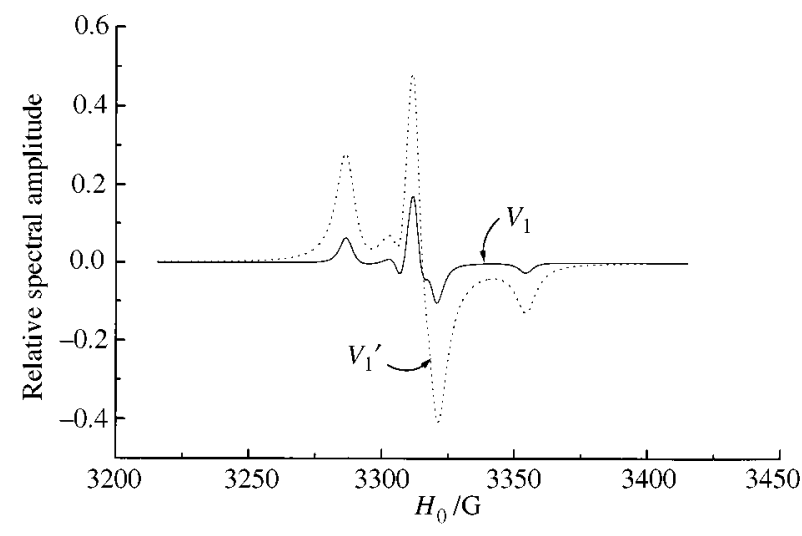

Fig. 3 Simulated first-harmonic absorption, in-phase (full line) and out-of-phase (broken line) EPR spectra for a rigid aminoxyl spin label. The simulations are for $T_{1}=2 \mu \mathrm{s}, T_{2}=20 \mathrm{~ns}, H_{1}=0.4 \mathrm{G}$ and $\omega_{\mathrm{m}} / 2 \pi=100$ $\mathrm{kHz}$.

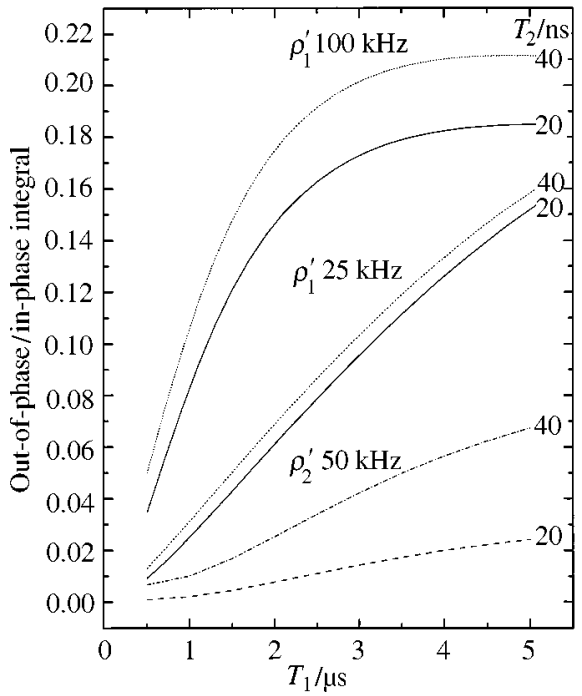

Fig. 4 Dependence of the ratio, $\rho_{1}{ }^{\prime}$, of the second integrals of the outof-phase to in-phase, first-harmonic absorption EPR spectra, on spinlattice relaxation time for a rigid aminoxyl spin label. The values of $T_{2}$ are indicated on the figure. The upper data for $\rho_{1}{ }^{\prime}$ are for a modulation frequency $\omega_{\mathrm{m}} / 2 \pi=100 \mathrm{kHz}$ and the lower data for $\rho_{1}{ }^{\prime}$ are for $\omega_{\mathrm{m}} / 2 \pi=25 \mathrm{kHz}$, with $H_{1}=0.5 \mathrm{G}$ for both. At the bottom of the figure, the ratio, $\rho_{2}{ }^{\prime}$, of the first integral of the out-of-phase, second-harmonic absorption EPR spectrum to the second integral of the in-phase, firstharmonic absorption EPR spectrum, with $H_{1}=0.25 \mathrm{G}$, is given for comparison.

of the first-harmonic absorption spectra resolves potential arbitrariness with regard to the choice of spectral position at which the spectral amplitude is measured. Simulated spectra of the inphase and out-of-phase first-harmonic absorption are compared in Fig. 3, for a rigid aminoxyl spin label, in the absence of molecular rotation. It is seen that the out-of-phase signal has very useable spectral intensities, especially in the case of high modulation frequencies. Eqns. (8) and (9) suggest ways in which calibrations of the normalised spectral intensities in terms of the dependences on $T_{1}$ and $T_{2}$ that are established by such lineshape simulations may be analysed.

It is found from lineshape simulations, that the dependence of the ratio, $\rho_{1}{ }^{\prime}$, of the second integrals of the out-of-phase to in-phase first-harmonic absorption spectra on $T_{1}$-relaxation time (Fig. 4) may be fitted empirically by the eqn. (10), where

$$
\rho_{1}^{\prime}=\frac{a_{1}{ }^{\prime} T_{1}^{m}}{1+b_{1}{ }^{\prime} T_{1}{ }^{m}}
$$

$a_{1}{ }^{\prime}, b_{1}{ }^{\prime}$ and the exponent $m$ are fitting parameters. A depend- 
ence of this form may be expected on the basis of eqn. (8) with $m=2$ for the spectral amplitudes. This is found by simulation to be the case for the spectral integrals with a modulation frequency of $\omega_{\mathrm{m}} / 2 \pi=100 \mathrm{kHz}$. For the lower modulation frequency of $\omega_{\mathrm{m}} / 2 \pi=25 \mathrm{kHz}$, the dependence of the ratio, $\rho_{1}{ }^{\prime}$, of spectral integrals on $T_{1}$ is better fitted with $m=1.3$. Also as expected, the coefficient $a_{1}{ }^{\prime}$ is only weakly dependent on $T_{2}$ (for normalization to the in-phase signal) but is directly dependent on the modulation frequency. For a modulation frequency of $100 \mathrm{kHz}$ and $T_{2}=20 \mathrm{~ns}, a_{1}{ }^{\prime} \approx 0.1$ and $b_{1}{ }^{\prime} \approx 0.7(m=2)$, in the absence of molecular rotational motion. At the lower modulation frequency $(25 \mathrm{kHz})$ and $T_{2}=20 \mathrm{~ns}, a_{1}{ }^{\prime} \approx 0.03$ and $b_{1}{ }^{\prime} \approx 0.07$ ( $m=1.3$ ), where in both cases $T_{1}$ is expressed in $\mu$ s in eqn. (10) and $H_{1}=0.5 \mathrm{G}$.

For the second-harmonic absorption spectra, it is found that the ratio, $\rho_{2}{ }^{\prime}$, of the first integral of the out-of-phase spectrum to the second integral of the first-harmonic in-phase spectrum (see Fig. 4) is fitted empirically by eqn. (11). This equation for

$$
\rho_{2}^{\prime}=\rho_{2}{ }^{0}+\frac{a_{2}{ }^{\prime} T_{1}^{2}}{1+b_{2}{ }^{\prime} T_{1}^{2}}
$$

the dependence of the normalised intensity on $T_{1}$ bears similarities to eqn. (9) where, in this case, $a_{2}{ }^{\prime}$ and $\rho_{2}{ }^{0}$ are directly dependent on $T_{2}$ and on the modulation frequency. In the absence of molecular motion, the calibration parameters obtained by spectral simulation are: $\rho_{2}{ }^{0} \approx 10^{-5}, a_{2}{ }^{\prime} \approx 4 \times 10^{-4}$ and $b_{2}{ }^{\prime} \approx 0.06$, for $T_{2}=20 \mathrm{~ns}, H_{1}=0.25 \mathrm{G}$ and a modulation frequency of $\omega_{\mathrm{m}} / 2 \pi=50 \mathrm{kHz}$. Again $T_{1}$ is expressed in $\mu \mathrm{s}$, for the calibration given by eqn. (11). These practical simulations illustrate how the non-linear EPR methods may be used to obtain values of $T_{1}$ and spin-lattice relaxation enhancements in real experimental situations.

\section{Conclusions}

The first- and second-harmonic absorption EPR signals detected $90^{\circ}$-out-of-phase with respect to the static field modulation are predicted to exhibit a practically useful sensitivity to spin-lattice relaxation that in many circumstances exceeds that of progressive saturation measurements. Whereas the firstharmonic non-linear EPR display is unsuitable for the study of slow rotational diffusion by conventional saturation transfer EPR spectroscopy, it provides a useful alternative for determin- ing $T_{1}$-relaxation enhancements of spin-labelled systems. The potential advantages of first-harmonic phase-quadrature absorption EPR, for the latter type of experiment, lie in the greater signal strength and comparative insensitivity to $T_{2}$ relaxation. Additionally, different regimes of $T_{1}$-sensitivity may be probed by using different modulation frequencies. Preliminary experimental support for the practicability of the firstharmonic display has been obtained with spin labels (Livshits, Páli and Marsh, unpublished). The usefulness of the secondharmonic display has already been demonstrated for spinlattice relaxation enhancements in which the $T_{2}$-relaxation is not changed appreciably.

\section{Acknowledgements}

This work was supported in part by the Deutsche Forchungsgemeinschaft (DFG).

\section{References}

1 D. Marsh, Appl. Magn. Reson., 1992, 3, 53.

2 D. Marsh, Chem. Soc. Rev., 1993, 22, 329.

3 D. Marsh, T. Páli and L. I. Horváth, in Spin-Labelling: The Next Millenium, ed. L. J. Berliner, Plenum Publishing Corporation, New York, 1997, in the press.

4 W. L. Hubbell and C. Altenbach, in Membrane Protein Structure: Experimental Approaches, ed. S. H. White, Oxford University Press, New York, 1994, p. 224.

5 D. D. Thomas, L. R. Dalton and J. S. Hyde, J. Chem. Phys., 1976, 65, 3006.

6 D. Marsh and L. I. Horváth, J. Magn. Reson., 1992, 97, 13.

7 T. Páli, V. A. Livshits and D. Marsh, J. Magn. Reson., Ser. B, 1966 , 113, 151.

8 K. Halbach, Helv. Phys. Acta, 1954, 27, 259.

9 D. D. Thomas and H. M. McConnell, Chem. Phys. Lett., 1974, 25, 470.

10 T. Páli, L. I. Horváth and D. Marsh, J. Magn. Reson., Ser. A, 1993, 101, 215

11 L. I. Horváth, P. J. Brophy and D. Marsh, Biophys. J., 1993, 64, 622.

12 L. I. Horváth and D. Marsh, J. Magn. Reson., 1983, 54, 363.

13 T. Páli, R. Bartucci, L. I. Horváth and D. Marsh, Biophys. J., 1992, 61, 1595.

14 T. Páli, R. Bartucci, L. I. Horváth and D. Marsh, Biophys. J., 1993, 64, 1781.

Paper 7/02476B

Received 10th April 1997

Accepted 2nd June 1997 